\title{
Characterization of Liquid Trickle Flow in Poor-Wetting Packed Bed
}

\author{
Shungo NATSUI, ${ }^{1 *}$ Tatsuya KIKUCHI, ${ }^{1)}$ Ryosuke O. SUZUKI, ${ }^{1)}$ Tatsuya $\mathrm{KON}^{2}{ }^{2)}$ Shigeru UEDA ${ }^{2)}$ and \\ Hiroshi NOGAMI ${ }^{2)}$
}

1) Division of Materials Science and Engineering, Faculty of Engineering, Hokkaido University, Kita 13-Nishi 8, Kita-ku, Sapporo, 060-8628 Japan.

2) Institute of Multidisciplinary Research for Advanced Materials (IMRAM), Tohoku University, 2-1-1 Katahira, Aoba-ku, Sendai, 980-8577 Japan.

(Received on August 11, 2014; accepted on December 24, 2014)

\begin{abstract}
A low coke rate operation of a blast furnace tends to cause deterioration of the gas permeability. The liquid iron and molten slag dripping under the melting zone influences the gas flow and permeability in the lower part of the furnace. A computational fluid dynamic model, using a particle-based simulation, is presented for characterization of the melt dripping behavior in a packed bed. In this work, the validity of the liquid passing conditions based on the gravity to surface tension ratio was confirmed. The melt shape produces an "icicle," "droplet," and "dome" forms based on the change of the surface tension and density absolute values. Even with an identical liquid volume passage, the form of the liquid flow changed by the pressure from the liquid's upper portion. Even if it has identical gravity and surface tension ratios, the liquid flow changes with the volume of the liquid phase and its form.
\end{abstract}

KEY WORDS: ironmaking; molten iron; trickle flow; packed bed; particle simulation.

\section{Introduction}

Reduction of $\mathrm{CO}_{2}$ emissions in the steel industry is an important global challenge, especially in the field of ironmaking, as strong trends are foreseen towards reducing blast furnace operations. In the ironmaking process, a low carbon operation is linked to this problem, and reducing the coke rate, which accounts for the larger amount of carbon input, is necessary. However, as a shift to a low coke rate operation will also reduce the amount of coke available to secure gas permeability, significant changes in the blast furnace's gas flow behavior will occur. In particular, as the gas flow permeability in the melting zone differs considerably from that in the lumpy zone, controlling this region has a large influence on the gas flow. Thus, an accurate understanding of the melt behavior characteristics is vital for the construction of a low carbon blast furnace. ${ }^{1)}$

Blast furnaces have both a cross (gas flow is perpendicular to the liquid flow) and counter-current (the gas and liquid flow are in opposing directions) flow conditions, shown in Fig. 1. The solids form a packed bed, while a two-phase solid-gas system exists in the upper furnace, and a threephase solid-gas-liquid system exists in the lower furnace. The liquid phase movement in the bottom furnace showed that the slag and molten iron formed in the cohesive zone and drip through a coke-packed bed to reach the furnace hearth. The flow of the liquid phase in this process is rec-

* Corresponding author: E-mail: natsui@eng.hokudai.ac.jp

DOI: http://dx.doi.org/10.2355/isijinternational.55.1259 ognized as an important phenomenon, as it influences the blast furnace productivity and operational stability, as well as the molten iron composition. Understanding the liquid holdup process, besides providing useful information on liquid residence times, would facilitate operation design. Abundant research is available related to liquid holdups in packed beds. Fukutake et l. $^{2)}$ have performed a comprehensive study on liquid holdups, while Usui et $a l^{3)}$ performed

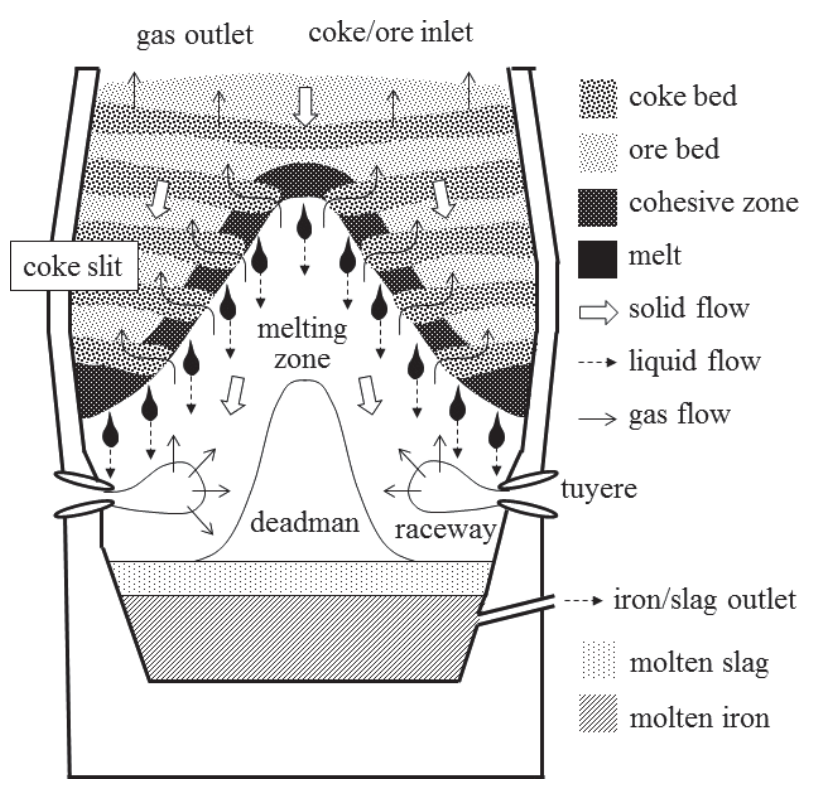

Fig. 1. Schematic diagram of the lower part of the blast furnace. 
a holdup study in the presence of air using both stationary and moving beds with a cold model experiment.

However, the conditions in metallurgical systems are quite different, as they are frequently non-wetting in nature, with a higher liquid density, and low superficial liquid velocity. Although the degree of holdup was related to the surface tension to gravity ratio based on a water model experiment, ${ }^{2,4-6)}$ there is a valuable report on the molten iron and slag holdup estimation by high temperature experiments. ${ }^{7)}$ However, few researchers have reported data on iron liquid holdup pertaining to metallurgy. ${ }^{8)}$ Such dripping phenomena are influenced by the liquid's physical properties, interfacial phenomena, and other physical properties. Understanding the dynamic behavior of a molten iron interface is problematic due to its dependence on a combination of several physical properties including density, viscosity, interfacial energy, velocity, and shape. Consequently, although many quantitative analyses have been attempted, it remains extremely difficult to accurately predict the molten iron interface shape through experimental data alone.

In order to clarify liquid holdups under discrete flow conditions, numerical models can enable a better understanding of these conditions, such as the force balance of the fluid. With the recent progress in computational science and technology, it is possible that a high-accuracy numerical simulation could provide an understanding of the molten iron interface transient behavior. Currently, the droplet behavior on the solid surface has been studied using a novel numerical particle simulation. ${ }^{9)}$ In this method, although the liquid's velocity on the solid was not always predicted, the surface tension's absolute value was correctly estimated. ${ }^{10)}$ The aims of this study are to clarify the melt dripping mechanism in the packed bed and to classify its interfacial shape using a 3-dimensional particle simulation.

\section{Methods}

\subsection{Calculation Procedure}

Using the particle method, the fluid flow is tracked using discrete particles in a Lagrangian system, wherein all liquids are assumed as incompressible due to a sufficiently small Mach number. The flow of each fluid phase is governed by the continuity equation and the Navier-Stokes equation for incompressible fluids:

$$
\begin{gathered}
\frac{D \rho}{D t}+\rho \nabla \cdot \mathbf{u}=0 \\
\rho \frac{D \mathbf{u}}{D t}=\mathbf{F}_{p}+\mathbf{F}_{v}+\mathbf{F}_{g}+\mathbf{F}_{s}
\end{gathered}
$$

where $\mathbf{u}$ is the velocity, $t$ is the time, $\rho$ is the fluid's density, $\mathbf{F}_{p}$ is the pressure term, $\mathbf{F}_{v}$ is the viscosity term, $\mathbf{F}_{g}$ is the gravity force, and $\mathbf{F}_{s}$ is the surface tension in terms of force per unit volume of fluid. Equation (2) is discretized by the moving particle semi-implicit (MPS) method, where the pressure gradient is calculated by an implicit method; whereas all other terms are calculated using an explicit method:

$$
\rho \frac{D \mathbf{u}}{D t}=\left[\mathbf{F}_{p}\right]^{t+\Delta t}+\left[\mathbf{F}_{v}\right]^{t}+\left[\mathbf{F}_{g}\right]^{t}+\left[\mathbf{F}_{s}\right]^{t}
$$

An explicit solution to this particle motion equation is obtained by substituting the differential operators in the governing equations into the MPS model:

$$
\mathbf{u}^{*}=\mathbf{u}^{t}+\frac{\Delta t}{\rho}\left[\mathbf{F}_{v}+\mathbf{F}_{g}+\mathbf{F}_{s}\right]^{t}
$$

where $\mathbf{u}^{*}$ is the velocity in the prediction step. The $\mathbf{F}_{p}$ remains an unknown value, therefore it is solved using the particle density in the prediction step, which in turn is explicitly solved using the particle velocity and initial particle density. A convergent calculation is applied to the pressure term instead of an explicit calculation to ensure interfacial stability, with the velocity of each particle then obtained using $\left[\mathbf{F}_{p}\right]^{t+\Delta t}$ :

$$
\mathbf{u}^{t+\Delta t}=\mathbf{u}^{*}+\frac{\Delta t}{\rho}\left[\mathbf{F}_{p}\right]^{t+\Delta t}
$$

We have already confirmed the total mass and volume preservation (over 99\%) with this numerical method. Detailed information on this calculation method is provided in previous articles. ${ }^{9,10)}$

\subsection{Calculation Conditions}

Calculations were performed for each packed bed system, with the calculation domain shown in Fig. 2, aimed at examining the free fall, collision with solids, and droplet

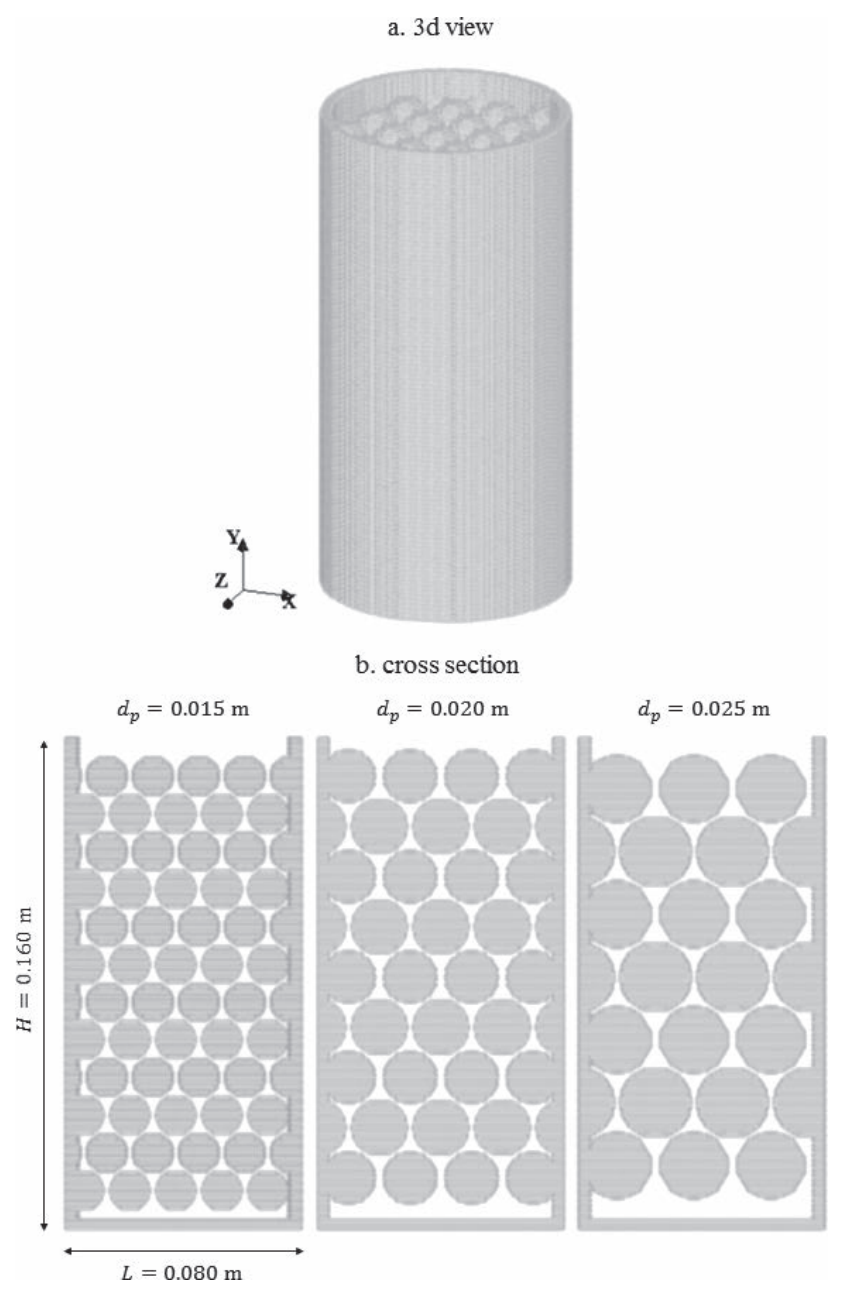

Fig. 2. Computational domain, with the solids filled by calculated element particles. 
modification in each packed bed. The container is a cylindrical shape with the packed solids embedded in the inner wall's surface. The melt's dynamic behavior was tracked by "melting" the top portion of arbitrary solids. As shown in this figure, we prepared a hexagonal close pack (HCP) structure (void fraction, $\varepsilon=0.26$ ) with different diameters of filler solids $\left(d_{p}=0.015,0.020\right.$, and $\left.0.025 \mathrm{~m}\right)$. The calculation conditions are shown in Table 1, with the particle diameter $\left(l_{0}\right)$ remaining constant. It should be noted that $l_{0}$ is not a solid filler diameter, but rather a calculated element particle diameter. The typical fluid physical properties used in this study were estimated ${ }^{2,8)}$ as shown in Table 2 . In this study, only solids with poor wettability were used. Therefore, the interfacial interaction force between the liquid and solid was zero, resulting in a $180^{\circ}$ contact angle $(\theta) .{ }^{9)}$ In this study, the

Table 1. Calculation conditions.

\begin{tabular}{rc}
\hline time step $\Delta t[\mathrm{~s}]$ & $1.0 \times 10^{-5}$ \\
diameter of particles $d_{0}[\mathrm{~m}]$ & $1.0 \times 10^{-3}$ \\
max particle number $N[-]$ & 800000 \\
\hline
\end{tabular}

Table 2. Liquid physical properties.

\begin{tabular}{rcc}
\hline Fluid & Water & Iron \\
\hline density $\rho\left[\mathrm{kg} / \mathrm{m}^{3}\right]$ & 1000 & 7000 \\
viscosity $\mu[\mathrm{Pa} \cdot \mathrm{s}]$ & $1.39 \times 10^{-3}$ & $7.00 \times 10^{-3}$ \\
surface tension $\sigma[\mathrm{N} / \mathrm{m}]$ & $7.28 \times 10^{-2}$ & 1.00 \\
\hline
\end{tabular}

Bond number is defined as the surface tension and gravity force ratio $\left(\mathrm{Bo}=\rho g d_{p}^{2} / \sigma\right)$. When $d_{p}=15 \mathrm{~mm}, \mathrm{Bo}=30.29$ in water and 15.44 in iron.

We then applied this simulation to a constant Bond number system in order to investigate the trickle flow changes. The melt's density and surface tension value were changed, with their Bo fixed to that of water $\left(\rho_{0}, \sigma_{0}\right)$. Here, we defined the coupled proportional coefficient as $\beta=\rho / \rho_{0}=\sigma / \sigma_{0}$. In this condition, a droplet's interface shape becomes uniform if the Bo number is constant, as it represents the ratio of the force of gravity to that of a surface. Additionally, in this numerical method, it has been confirmed that the liquid droplet's shape on a flat solid does not change under a constant Bo number as water condition. Here, we set $\mu=\beta \mu_{0}$ in order to maintain a constant Reynolds number $\left(\operatorname{Re}=\rho v d_{p} / \mu\right)$.

The programs used in this research were designed by the authors in Fortran90/95 and compiled using Intel ${ }^{\circledR}$ Fortran Composer, Version 14.1. The CPUs used in this work were Intel ${ }^{\circledR}$ Core i7-4 $820 \mathrm{~K}(3.7 \mathrm{GHz}, 4$ cores $) \times 9$.

\section{Results and Discussion}

\subsection{The Behavior of Water and Iron Droplets in Packed Beds}

The liquid phase frequently utilized in a cold model experiment is room temperature water. In this section, the water's behavior in a packed bed is considered. The calculated results of the shape change in a single droplet moving through a packed bed over time are shown in Fig. 3. The packed solids are not shown, only the liquid phase.
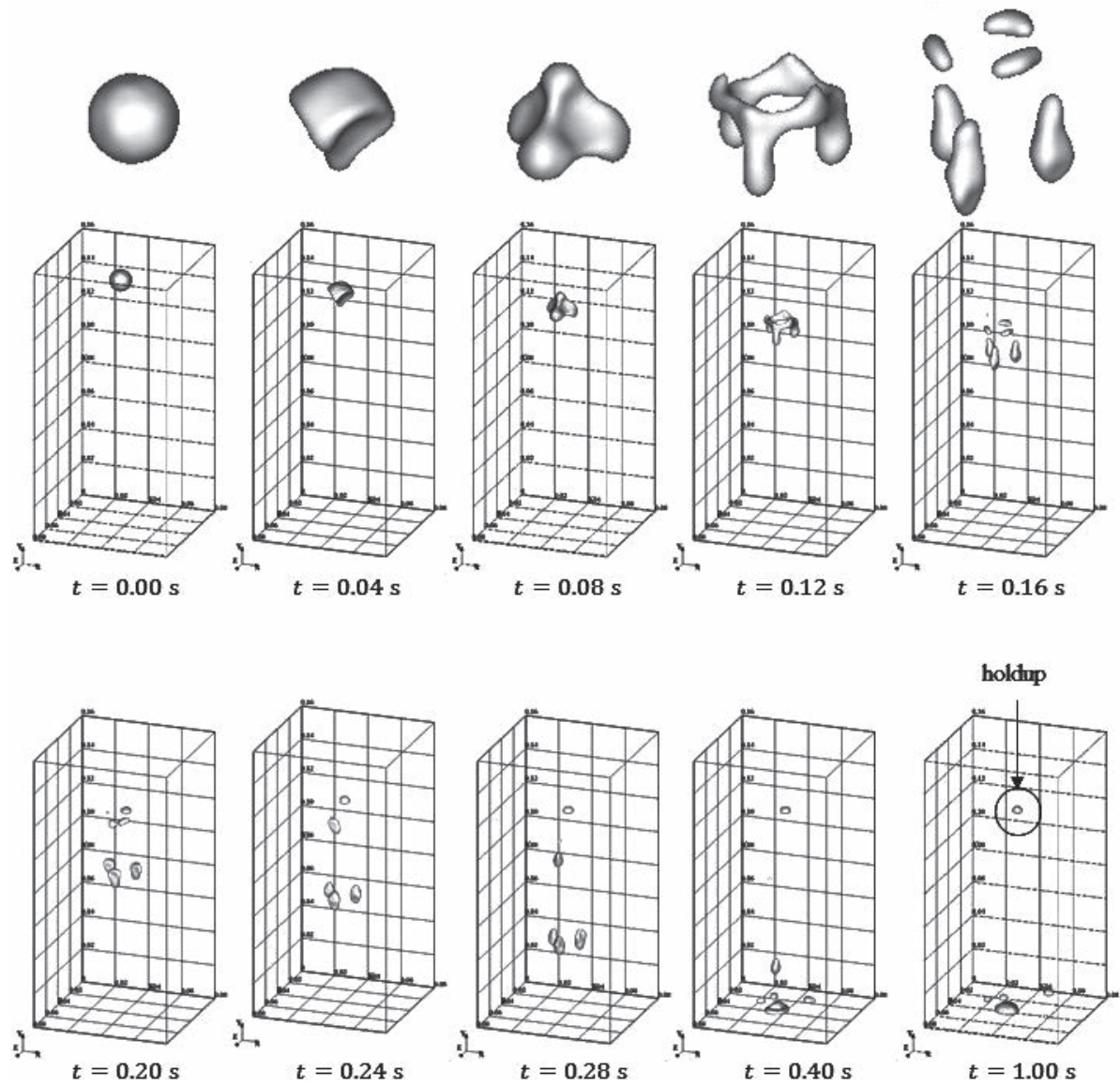

Fig. 3. The calculated shape change in a single water droplet progressing through a packed bed $\left(d_{p}=0.015 \mathrm{~m}\right)$ over time. 
The initial diameter of the droplet is $d_{p}=15 \mathrm{~mm}$. As the droplet fell due to gravity, it collided with the solids and trickled into the bed. From $\mathrm{t}=0.12$ to $0.16 \mathrm{~s}$, a single droplet dispersed into small droplets. This trickling behavior is in agreement with experimental results. ${ }^{6}$ Although the amount of static hold up is estimated to be zero when $\theta=180^{\circ}$ (based on non-dimensional analysis), ${ }^{2)}$ part of the droplet remains above the packed solid. This may be the result of the numerical discretization process. Calculation accuracy is expected as below. The influence radius which calculate the surface tension of each surface particle was set to $2.3 d_{p}$ in this particle simulation. ${ }^{10)}$ Particle diameter $d_{p}$ is fixed as $1.0 \mathrm{~mm}$, so the calculation accuracy is guaranteed around 3 particles at the surface, i.e., $3.0 \mathrm{~mm}$ will be the minimum value of droplet diameter. Droplet of less than $3.0 \mathrm{~mm}$ of diameter will drop like a rigid body ball. On the other hand, dispersed small droplets were larger than $3.0 \mathrm{~mm}$ in this case. Therefore, calculation resolution is guaranteed in this calculation condition.

The influence of the droplets in the radial direction was also considered. The progress of two droplets on a same horizontal plane in a HCP structure was then calculated. The calculated result of the shape change in the two water droplets as they trickled down the inside of a packed bed is shown in Fig. 4. In Fig. 4(a), since the distance between the two droplets is minimal, they came into contact at approximately $\mathrm{t}=0.15 \mathrm{~s}$. Due to this contact, they divided into a long icicle shape with some small droplets as they trickled down the packed bed. On the other hand, in Figs. 4(b) and 4(c), although the droplets fell independently without a collision in either case, only c) showed a static hold up similar to Fig. 3. This may be from the influence of the packed bed structure. Even with a droplet of the same volume, the dripping behavior may change based on the bed structure.

The droplet behavior of molten iron was then considered. The shape change in a single droplet of molten iron over time is shown in Fig. 5. The molten iron's change is quite different from water. A single iron droplet did not have a change in its interface shape, and it was completely held up above the top of the packed bed. The Bo number of iron is half that of water and its surface tension is the dominant force, rather than gravity. As the surface tension is greater
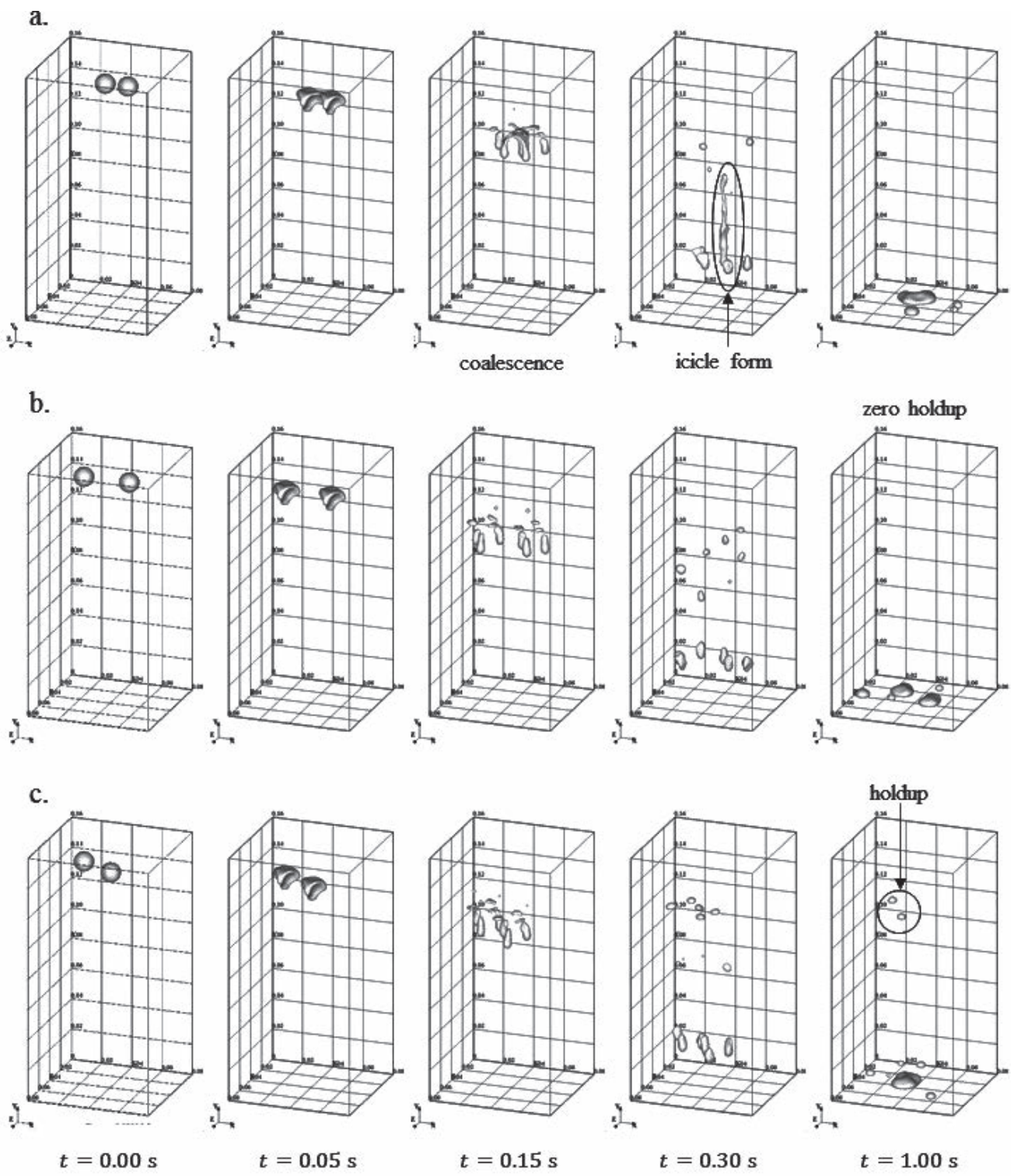

Fig. 4. The calculated shape change in two water droplets for each arrangement ( $d_{p}=0.015 \mathrm{~m}$ ) over time (a. neighboring, b. alternate, c. diagonal). 


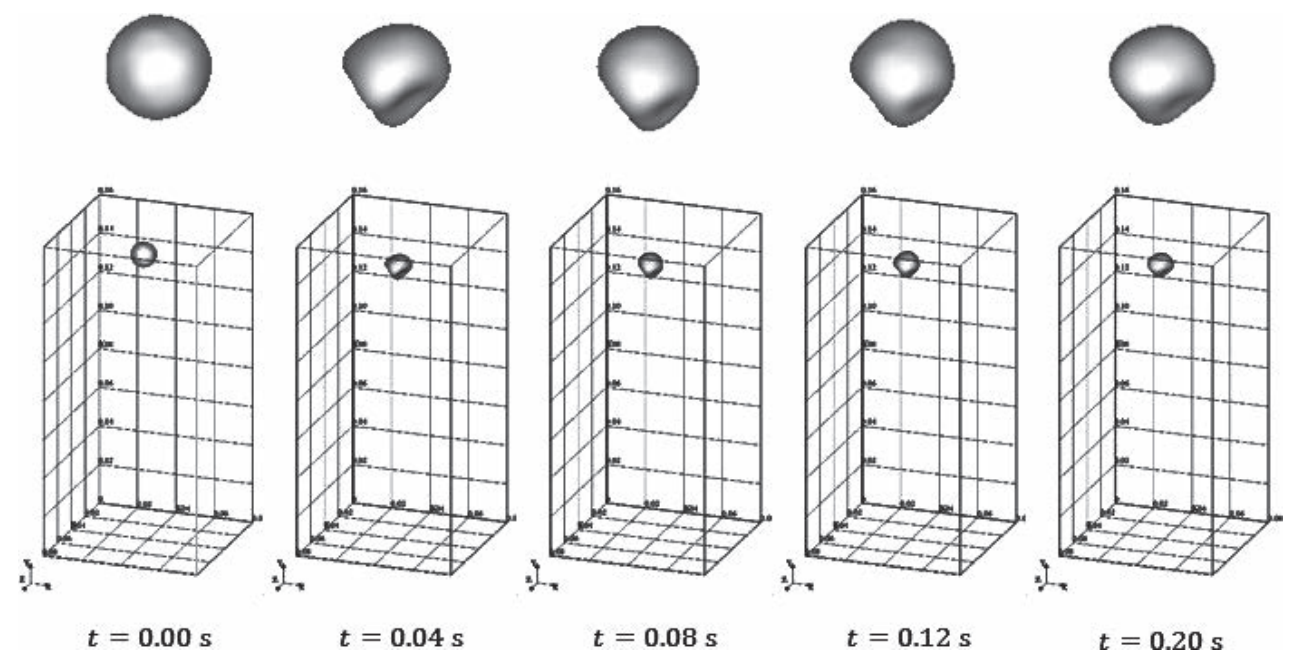

Fig. 5. The calculated shape change of a single iron droplet through a packed bed $\left(d_{p}=0.015 \mathrm{~m}\right)$ over time.
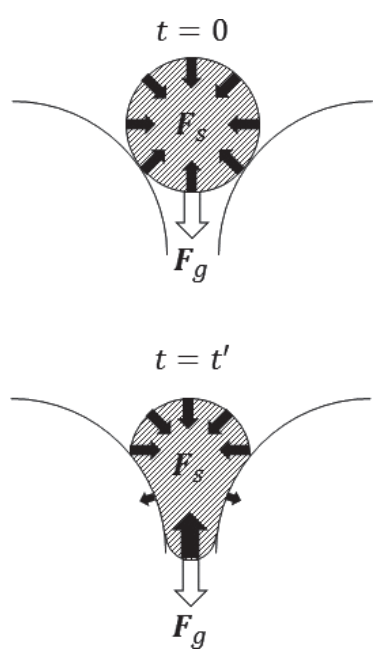

Fig. 6. The force balance of a droplet moving between packed particles.

than gravity, which is the driving force of a droplet moving down, it cannot pass through the voids in the packed bed. The force balance between the droplet and solid surfaces is shown in Fig. 6. The wettability was completely ignored. At $t=0 \mathrm{~s}$, the attractive force towards the center of the droplet maintains its spherical shape. At $t=t^{\prime}$, the droplet collides with the solid surfaces, changing shape and filling the void. The absolute value of the force from the surfaces will change with the curvature of the liquid's lower portion.

$$
\mathbf{F}_{s}=\sigma \kappa \mathbf{n}
$$

Here, $\kappa$ is the liquid's curvature, and $\mathbf{n}$ is the unit vector normal to the surface. $\kappa$ is described as: $\kappa=1 / R$ with $R$ as the curvature radius. In the case of a single droplet, the dripping can be described by the force balance in the vertical direction, i.e., the balance of $\mathbf{F}_{g}$ to $\mathbf{F}_{s}$. The capillary length, $\lambda=\sqrt{\sigma / \rho g}^{11)}$ is one characteristic length scale for an interface subject to both gravitational acceleration and a surface force; its value is $2.73 \times 10^{-3} \mathrm{~m}$ in water and $3.82 \times 10^{-3} \mathrm{~m}$ in iron. In comparison, when the HCP packed bed consisted of solid spheres $0.015 \mathrm{~m}$ in diameter, the maximum diameter of an interstitial sphere was $d_{c}=0.224 d_{p}$, thus $d_{c}=3.36 \times 10^{-3}$ $\mathrm{m}$. This value is halfway between the water and iron capil- lary lengths. Therefore, in the case of a single droplet combined with a HCP packed bed, the dripping condition can be estimated by the following equation:

$$
d_{p}>\frac{1}{0.224} \sqrt{\frac{\sigma}{\rho g}}
$$

\subsection{Influence of Surface Tension and Density on the Trickle Flow}

In this section, we discuss the trickle flow behavior at a constant Bo number. The liquid physical property used is based on water, and the absolute values of the density and surface tension was multiplied by $\beta$. The interface shape of a single droplet and multiple droplets (two layers of top melting) at $t=0.25 \mathrm{~s}$ is shown in Fig. 7. In the case of a single droplet, it trickled down when $\beta=1$ and 2 , and it represented a static hold up when $\beta \geq 4$. When $\beta=4$, although the droplet's interface changed shape, a solid below it halts the liquid. When $\beta \geq 8$, the droplet remained between solid particles at the top of the packed bed.

Although all of these conditions are multiple constants of water's Bo numbers, the resultant droplet behavior differed. Based on this, their differences cannot be explained only by Eq. (7). On the other hand, for the multiple droplet conditions, they dripped as an icicle shape when $\beta=1$ and 2; a channel flow appeared when $\beta=4$, and it stagnated at the top of the bed as a dome shape when $\beta \geq 8$. As shown for $\beta=4$, the dripping behavior depends on the liquid volume (or height). The influence of the filling solid diameter on the dripping behavior is shown in Fig. 8. In this case, liquids drop in an icicle shape even if $\beta=8$. When each droplet has a similar Bo number and volume, the free surfaces become identical even if they have a different $\beta$ value. However, when the droplet shape is determined by the packed bed's void size, the change in the $\beta$ value influences the ratio of the $\mathbf{F}_{g}$ and $\mathbf{F}_{s}$.

From these results, the liquid dripping condition can be estimated based on the gravity and surface tension ratio per unit volume: ${ }^{11)}$

$$
d_{c}>\frac{2 \sigma}{\rho g h}
$$



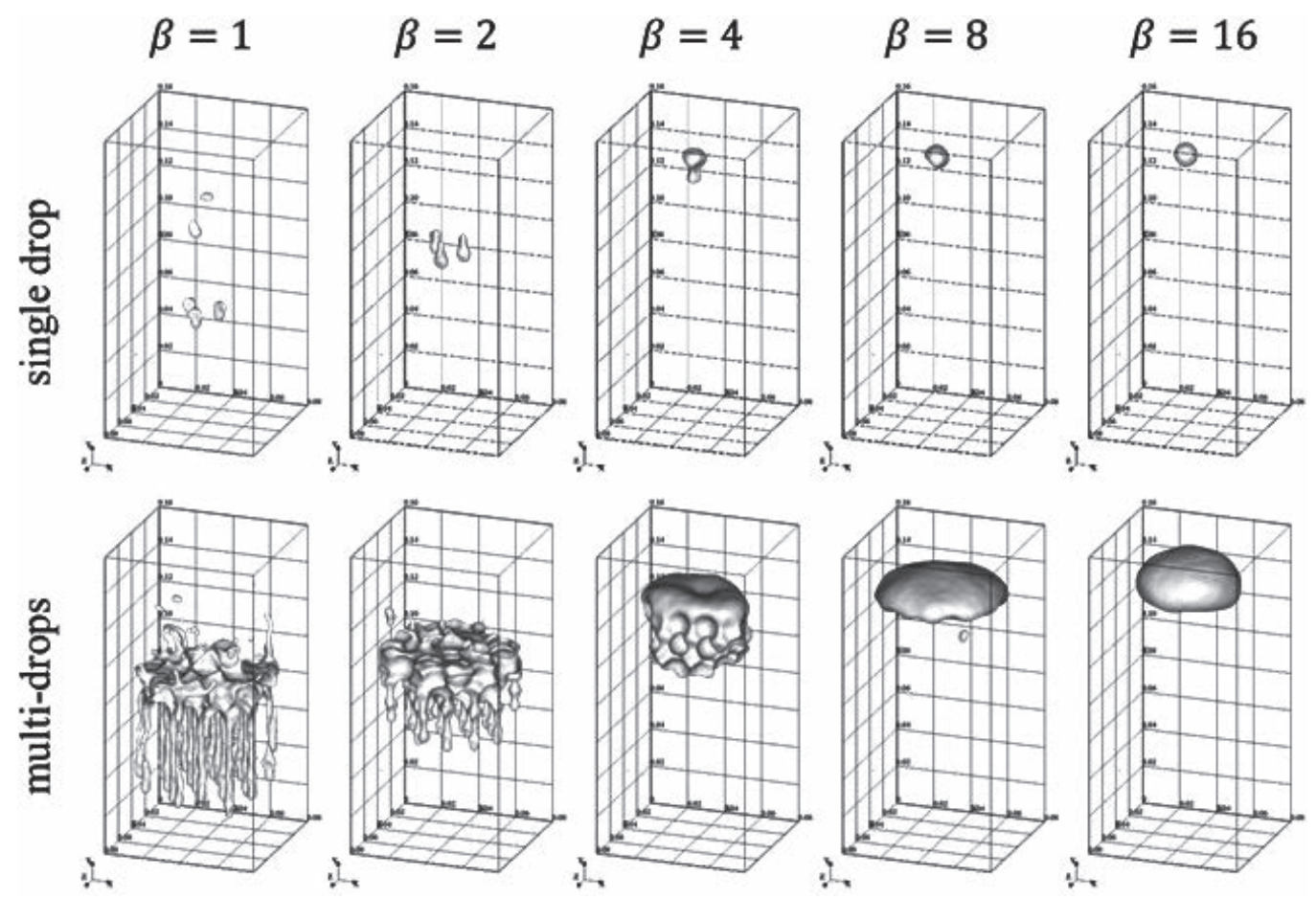

Fig. 7. The liquid interface shape snapshots at $\mathrm{t}=0.25 \mathrm{~s}\left(\mathrm{Bo}=30.29, d_{p}=0.015 \mathrm{~m}\right)$.
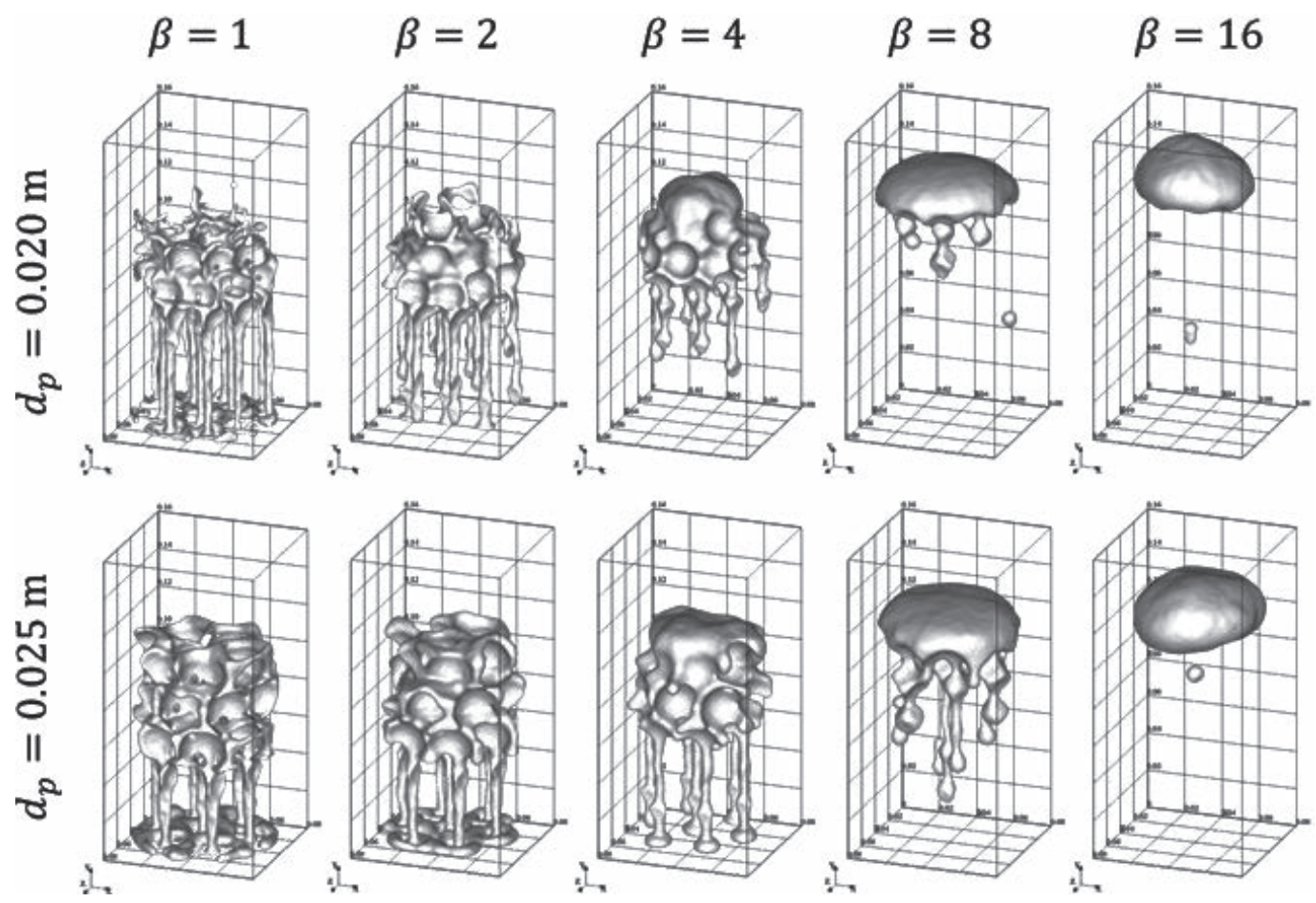

Fig. 8. The liquid interface shape snapshots at $\mathrm{t}=0.25 \mathrm{~s}\left(\mathrm{Bo}=30.29, d_{p}=0.020,0.025 \mathrm{~m}\right)$.

However, in order to also evaluate the modification energy of a droplet in the void between solids, the absolute value of $V$ must be determined. Here, even if droplets have similar Bo numbers, their dripping behavior will change based on the volume of the liquid.

\subsection{Classification of the Melt Behavior in a Poor-wet- ting Packed Bed}

The influence of $\beta$ and $V$ on the liquid trickle flow behavior is schematically shown in Fig. 9. If $V$ is sufficiently smaller than $d_{c}$ (i.e., the droplet is smaller than $\lambda$ ), then $\beta$ will not have an influence, but the droplet will trickle lower. On a droplet scale greater than $\lambda$, the droplet must deform in order to pass through a void between the solids. If $\beta$ becomes larger, the droplet cannot deform sufficiently to pass, thus it will stagnate on top of the packed solids. However, if $V$ increases, the liquid will be pushed through by hydrostatic pressure.

Due to the dispersion of the localized liquid, the classification of a flow behavior remains complicated. The 


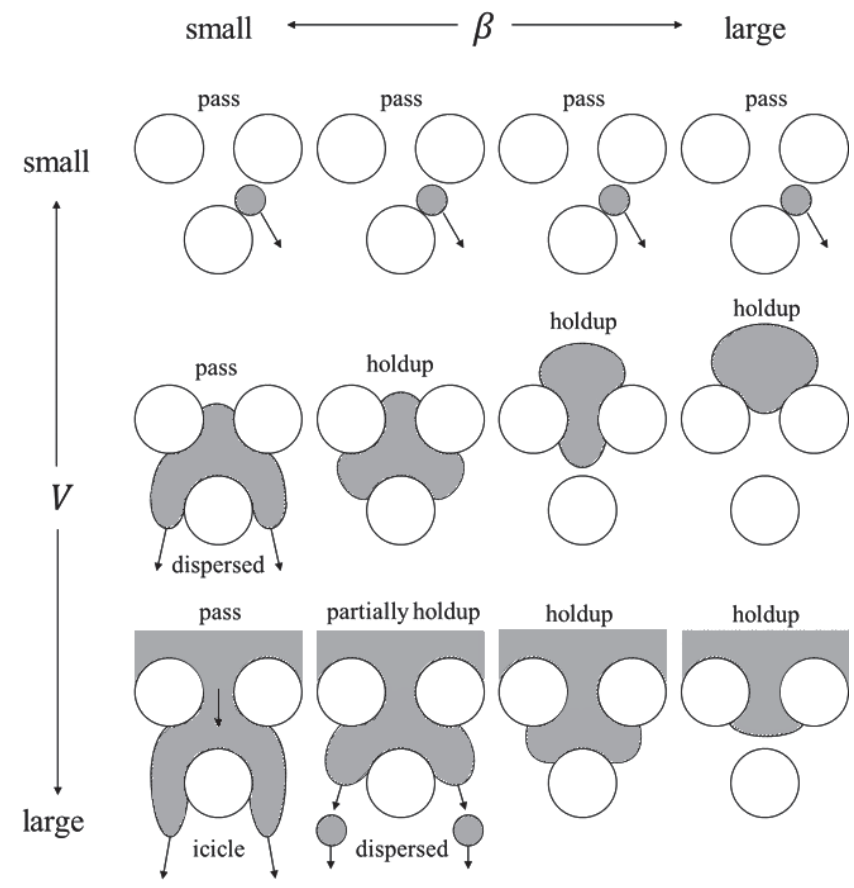

Fig. 9. The change in the liquid flow behavior based on its physical properties and volume in a poor-wetting packed bed.

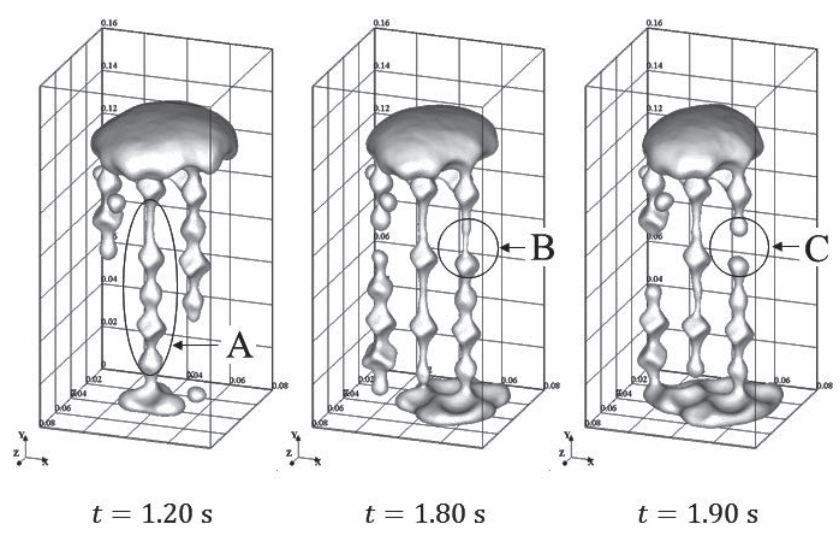

Fig. 10. The shape change in the calculated liquid interface through a packed bed $\left(\mathrm{Bo}=30.29, \beta=8, d_{p}=0.020 \mathrm{~m}\right)$.

change of the liquid interface when $\beta=8$, and $d_{p}=0.020 \mathrm{~m}$ over time is shown in Fig. 10. Figure 10(A) represents the liquid's icicle shape, and this dripped continuously without changing its free surface shape. In this case, the dimensionless analysis shown in the Appendix is applicable. However, as shown in Figs. 10(B) and 10(C), this icicle will elongate and separate when the pressure from the upper portion decreases. This demonstrates that the trickle flow behavior changes drastically even with similar physical properties. The holdup conditions for a packed bed should not only be derived as identical to a syringe-type injection, but the droplets' shape change should also be considered.

\section{Conclusions}

The liquid trickle flow behavior in a packed bed was numerically simulated in order to characterize molten iron dripping in the lower portion of a blast furnace. In this study, the wettability was assumed to be zero, and a con- stant solid diameter and HCP structure were introduced. As the density and surface tension values of the water model experiment differed greatly from actual molten iron, we performed the simulation across a wide range of physical properties. The following results were obtained:

(1) In the case of a single droplet with the same diameter as the packed solids, the dripping conditions based on the gravity to surface tension ratio can be applied. The maximum diameter of the voids must be maintained as:

$$
d_{c}>\sqrt{\frac{\sigma}{\rho g}}
$$

A molten iron droplet may be suitably small in the lower portion of the blast furnace, allowing it to pass through the packed bed when.

(2) When the liquid volume was larger than the packed solid diameter, the liquid shape will change based on the absolute value of the surface tension and density. The liquid shape showed an icicle, droplet, and dome shape, with increasing of absolute value of surface tension, respectively. In this case, the following dripping conditions can be applied, considering a dynamic balance between the localized gravity and surface tension:

$$
d_{c}>\frac{2 \sigma}{\rho g h}
$$

From this equation, the liquid dripping behavior is influenced by the pressure from the upper portion of the liquid, breaking the icicle shape due to its volume change, even with similar physical properties.

(3) Even if the same liquid volume is injected, the droplets represent different trickle flow behavior by coalescing or dispersing, changing the static hold up.

\section{Acknowledgments}

S. Natsui was partially supported by the Iron and Steel Institute of Japan (ISIJ) Research Promotion Grant. The authors would like to thank Mr. Hifumi Takai and Mr. Ryota Nashimoto from Hokkaido University for their supports in building a computer system.

\section{Nomenclature}

Symbols:
$d$ : diameter, $\mathrm{m}$
F: force, $\mathrm{N}$
g: gravity, $\mathrm{m} / \mathrm{s}^{2}$
$h$ : height, $\mathrm{m}$
$k$ : coefficient, -
$t$ : time, $\mathrm{s}$
u: velocity, $\mathrm{m} / \mathrm{s}$
$V$ : volume, $\mathrm{m}^{3}$

Greek letters:

$\beta$ : proportional coefficient of surface tension and density, -

$\mu$ : viscosity coefficient, $\mathrm{m}^{2} / \mathrm{s}$

$\rho:$ density, $\mathrm{kg} / \mathrm{m}^{3}$

$\sigma:$ surface tension coefficient, $\mathrm{N} / \mathrm{m}$

$\theta$ : contact angle, $^{\circ}$ 
Subscripts:
c: critical
g: gravity
$p$ : pressure
$s:$ surface
$v$ : viscosity

\section{REFERENCES}

1) M. Hayashi, S. Sukenaga, K. Ohno, S. Ueda, K. Sunahara and N. Saito: Tetsu-to-Hagané, 100 (2014), 211.

2) T. Fukutake and V. Rajakumar: Tetsu-to-Hagané, 66 (1980), 1937.

3) T. Usui, K. Masamori, H. Kawabata and Z. Morita: ISIJ Int., 33 (1993), 687.

4) G. S. Gupta and S. Bhattacharyya: ISIJ Int., 43 (2003), 1927.

5) T. Sugiyama, T. Nakagawa, H. Sibaike and Y. Oda: Tetsu-to-Hagané, 73 (1987), 2044.

6) K. Nishioka, D. Fujiwara, K. Ohno, T. Maeda and M. Shimizu: ISIJ Int., 50 (2010), 1016.

7) H. Ohgusu, Y. Sassa, Y. Tomita, K. Tanaka and M. Hasegawa: Tetsuto-Hagané, 78 (1992), 1164.

8) I. Jeong, H. Kim and Y. Sasaki: ISIJ Int., 53 (2013), 2090.

9) T. Kon, S. Natsui, S. Ueda, R. Inoue and T. Ariyama: ISIJ Int., 53 (2013), 590.

10) S. Natsui, R. Soda, T. Kon, S. Ueda, J. Kano, R. Inoue and T. Ariyama: Mater. Trans., 53 (2012), 662.

11) P. de Gennes, F. Brochard-Wyart and D. Quéré: Capillarity and Wetting Phenomena: Drops, Bubbles, Pearls, Waves, Yosioka Shoten, Kyoto, (2003), 32.

12) E. Buckingham: Phys. Rev., 4 (1914), 345.

\section{Appendix}

Figure 11 shows the system with a liquid column of a height $(h)$, and a poor-wetting solid with a columnar hole with a diameter $\left(d_{c}\right)$. The following five physical quantities are assumed to be linked by the relation:

$$
f\left(h, d_{c}, \sigma, \rho, g\right)=0
$$

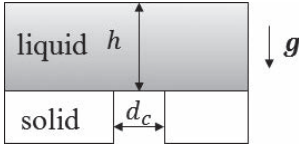

Fig. 11. Schematic of the liquid phase in contact with a solid having a columnar hole.

Thus, it can be written using a proportional constant $(k)$ and index numbers as follows:

$$
h=k d_{c}^{A} \sigma^{B} \rho^{C} g^{D}
$$

Using the following three basic dimensions, length $[L]$, time $[T]$, and mass $[M]$, we derived following dimensional formula:

$$
\begin{aligned}
{[L] } & =\left[L^{A}\right]\left[M^{B} T^{-2 B}\right]\left[M^{C} L^{-3 C}\right]\left[L^{D} T^{-2 D}\right] \\
& =\left[\mathrm{L}^{A-3 C+D} \cdot M^{B+C} \cdot T^{-2 B-2 D}\right]
\end{aligned}
$$

Here, the index number $(n)$ is used as:

$$
h=k d_{c}^{1+2 n} \sigma^{-n} \rho^{n} g^{n}
$$

Using Buckingham's $\Pi$ theorem, ${ }^{12)}$ we derived the relation of two dimensionless numbers:

$$
\frac{h}{d_{c}}=k\left(\frac{d_{c}^{2} \rho g}{\sigma}\right)^{n}=k \mathrm{Bo}^{n}
$$

Therefore, if the liquid phase trickles continuously (such as an icicle shape), the following dripping condition can be applied:

$$
h>k d_{c} \mathrm{Bo}^{n}
$$

